\title{
Maintenance Decision Based on Data Fusion of Aero Engines
}

\author{
Huawei Wang, ${ }^{1}$ Jun Gao, ${ }^{2}$ and Zhiyong Liu' ${ }^{2}$ \\ ${ }^{1}$ College of Civil Aviation, Nanjing University of Aeronautics and Astronautics, Nanjing 210016, China \\ ${ }^{2}$ Department of Management, Shijiazhuang Mechanical Engineering College, Shijiazhuang 050003, China
}

Correspondence should be addressed to Huawei Wang; huawei678@163.com

Received 18 January 2013; Accepted 4 April 2013

Academic Editor: H. K. Leung

Copyright (C) 2013 Huawei Wang et al. This is an open access article distributed under the Creative Commons Attribution License, which permits unrestricted use, distribution, and reproduction in any medium, provided the original work is properly cited.

\begin{abstract}
Maintenance has gained a great importance as a support function for ensuring aero engine reliability and availability. Costeffectiveness and risk control are two basic criteria for accurate maintenance. Given that aero engines have much condition monitoring data, this paper presents a new condition-based maintenance decision system that employs data fusion for improving accuracy of reliability evaluation. Bayesian linear model has been applied, so that the performance degradation evaluation of aero engines could be realized. A reliability evaluation model has been presented based on gamma process, which achieves the accurate evaluation by information fusion. In reliability evaluation model, the shape parameter is estimated by the performance degradation evaluation result, and the scale parameter is estimated by failure, inspection, and repair information. What is more, with such reliability evaluation as input variables and by using particle swarm optimization (PSO), a stochastic optimization of maintenance decision for aircraft engines has been presented, in which the effectiveness and the accuracy are demonstrated by a numerical example.
\end{abstract}

\section{Introduction}

Engines are the heart of an aircraft, whose failures can effect aircraft safety and lead to heavy economic losses. For aero engines, the most advanced preventive maintenance (PM) policy strategies rely on the monitoring of measurable parameters and making maintenance decisions on the level of the degradation of the system. Condition-based maintenance (CBM) has been used for aero engines, which has been proved useful in minimizing the cost of maintenance, improving operational safety, and reducing the quantity and severity of in-service failures. However, the traditional CBM only carries out maintenance tasks that focus on condition monitoring and diagnostics, which attempts to avoid unnecessary maintenance tasks when there is evidence of abnormal behaviors [1]. In recent years, $\mathrm{CBM}$ plus $(\mathrm{CBM}+)$, a development of CBM, has been put forward, which is the application and integration of appropriate process, technologies, and knowledgebased capabilities to improve reliability and maintenance effectiveness [2]. CBM+ can be viewed as cost-effective and accurate maintenance, which shows increasing importance in improving aero engines availability, reducing downtime cost, and enhancing operation reliability.

The core factors of maintenance decision can be summarized as condition monitoring, reliability evaluation, and decision optimization. Condition monitoring involves comparing online and offline data with expected values. Reliability evaluation and prediction are based on condition monitoring.

For aero engines, there is little failure data. However, additional information, including on-board sensor measurements, maintenance histories, and component data, is available. The goal of $\mathrm{CBM}+$ is to achieve more accurate maintenance. In order to achieve accurate maintenance, data fusion techniques are suggested. Thus, it is beneficial to put event data and condition monitoring data together. This combined data analysis can be accomplished by building a mathematical model that properly describes the underlying mechanism of a fault or a failure.

Applying fusion techniques in CBM has been receiving increasing attention. Niu et al. [3] employed data fusion strategy for improving condition monitoring, health assessment, 
and prognostics. A time-dependent proportional hazards model (PHM), which is a popular model in survival analysis, is suitable for analyzing both event and condition monitoring data. Jardine et al. [4] proposed using Weibull PHM to analyze the aircraft and marine engine failure data together with the metal concentration measurements of the engine oil. An extension of PHM is the proportional intensity model (PIM), which adopts a stochastic process and assumes a similar form to intensity function of the stochastic process. Vlok et al. [5] studied the application of PIM to analyze failure and diagnostic measurement data from bearings. Hidden Markov model (HMM) is another appropriate model for analyzing event and condition monitoring together. Bunks et al. [6] applied an HMM to analyze the Westland helicopter data which consists of gearbox fault class information and vibration measurements with different faults. Dong and $\mathrm{He}$ [7] proposed a more general model, hidden semi-Markov model, for analyzing pump experimental data in pump diagnostics and prognostics. Lin and Makis [8] proposed a partially observable stochastic model to describe the underlying failure mechanism of a system undergoing condition monitoring. Other models that appeared in the literature and that can be used to analyze both event and condition monitoring data are models using delay-time concept and stochastic process [9].

Maintenance decision actually is a problem of optimization [10]. Because of high safety demand for aero engines, the uncertainties must be taken into consideration. Given the cost of the monitoring and inspection, the uncertainties in the reliability evaluation and maintenance decision can be reduced by condition monitoring and inspection, but such uncertainties still exist. So when making maintenance decisions, one must pay enough attention to these uncertainties. The problem of uncertainties in maintenance decision has drawn attention in the field of reliability engineering. In consideration of parametric uncertainty and analyzing the uncertainties on the maintenance optimization, a mixed integer nonlinear optimization model had been proposed [11]. Aven and Castro [12] proposed a minimal repair replacement model with two types of failure and a safety constraint. Vatn and Aven [13] presented a framework for how to carry out the maintenance optimization in a practical decision making, which took the uncertainties into account. Sanchez et al. [14] addressed the problem of testing and maintenance optimization based on unavailability and cost criteria and considered epistemic uncertainty in the imperfect maintenance modeling.

The complexity of maintenance decision on aero engines can be described as follows.

First, there is degradation failure as well as sudden failure in aero engines, and they interact with each other.

Second, aero engines have little failure data and much condition monitoring information. The reliability evaluation can only be realized by drawing valuable reliability information from condition monitoring information.

Third, there are randomness and noise in the condition monitoring information. The utilization of condition monitoring information determines the accuracy of reliability evaluation.
Forth, the reliability variation of aero engines is stochastic, which added the difficulty for maintenance optimization.

The paper develops a new maintenance decision model that integrates data fusion. The remaining parts of the paper are organized as follows. Section 2 presents the framework of maintenance decision for aero engines based on information fusion. Section 3 gives a reliability evaluation model based on information fusion for aero engines. Section 4 puts forward a maintenance decision model for aero engines. In Section 5, a numerical example is provided to demonstrate the effectiveness and accuracy of the method. Finally, some concluding remarks are made.

\section{The Framework of Maintenance Decision for Aero Engines Based on Information Fusion}

The paper intends to combine recent research results concerning maintenance decision with reliability evaluation based on information and to propose the maintenance decision methods based on information fusion for aero engines. Its characteristics are reflected in the following aspects. First, it takes into account both the characteristics of information and failure mechanism and establishes the information fusion model, respectively, for condition monitoring information and event information. Second, the reliability evaluation model is based on gamma stochastic process, which utilizes condition monitoring information and event information together by shape and scale parameters estimation. Third, considering the uncertainties and cost optimization, the maintenance decision model is presented. The modeling process is shown in Figure 1.

\section{Reliability Evaluation Model Based on Data Fusion for Aero Engines}

3.1. Reliability-Related Information of Aero Engines. The condition monitoring parameters of aero engines can be described as follows.

(a) Gas path monitoring parameters. The key of aero engines is the gas path system, which consists of air compressor, combustor and turbine, and so forth. Gas path measurement consists of some subsets of interstage pressures and temperatures, spool speeds, and fuel flow. The key parameters of gas path measurements include exhaust gas temperature (EGT) and fuel flow (FF).

(b) Oil monitoring parameters. Oil monitoring parameters consist of various oil system temperatures, pressures, fuel temperature, and delivery pressure. Oil measurements are the auxiliary instruments for aero engines, which can be used for monitoring components of lubrication system and its sealing. The key parameters of oil measurements include oil pressure $(\mathrm{OP})$, oil temperature, and oil consumption rate $(\mathrm{OCP})$. 


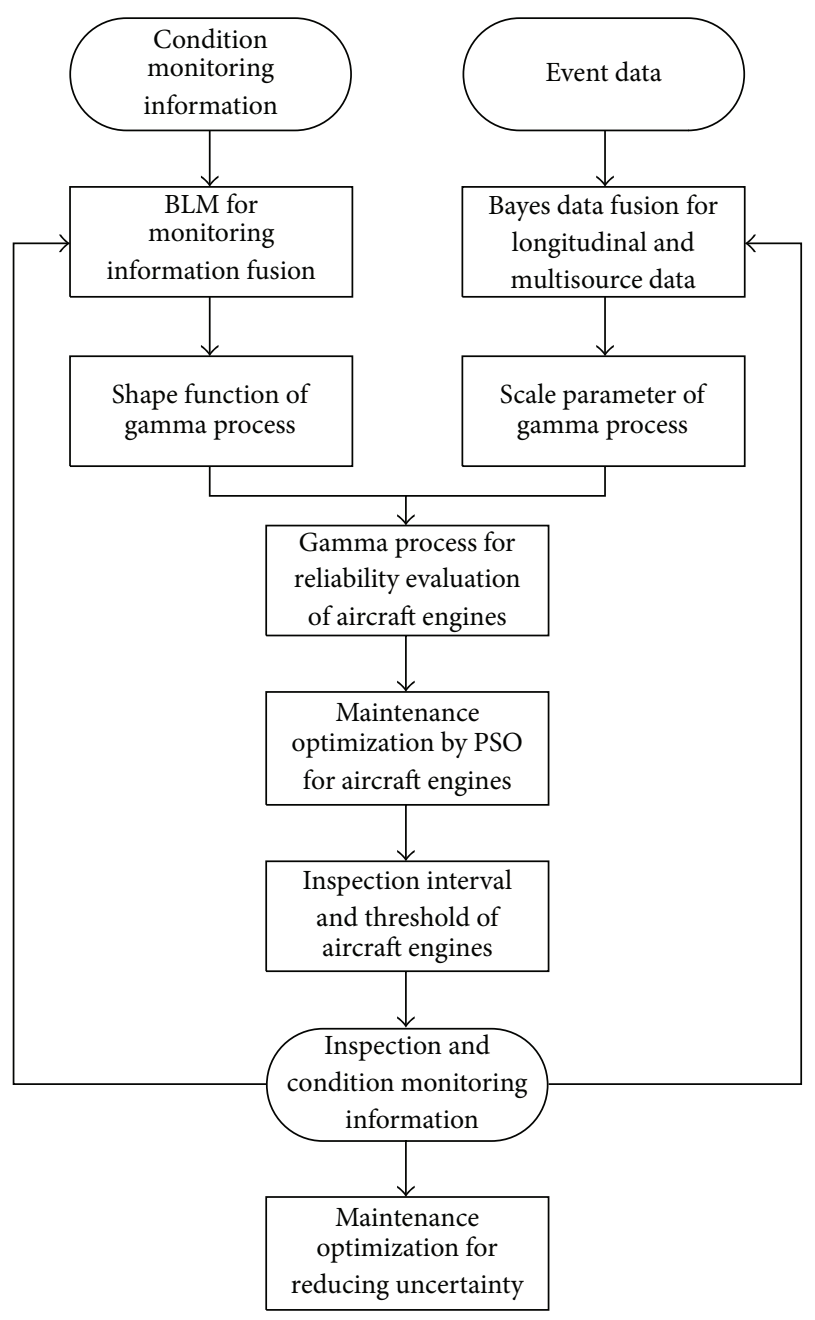

FIGURE 1: The flow diagram of maintenance decision for aero engines based on information fusion.

(c) Vibration measurements. High and low spools of aero engines are composed of blades, plates, axis, and bearings. There are some vibration signals while wear and damage occur during rotation. The key parameters of vibration measurements include low pressure vibration (LPV) and high pressure vibration (HPV).

The performance degradation is usually reflected on the change of condition monitoring parameters. For example, if EGT exceeds the standard, FF and OCP will increase, or high pressure rotor speed deviation (HPRSD) will occur, and a conclusion can be drawn that the aero engine is deteriorating.

Event data includes failure data and maintenance data. If there is enough failure data in event data, reliability can be evaluated directly. If not, event data can be used as prior information to support reliability evaluation. Similarly, knowledge of past maintenance and failure may be also used to aid in reliability evaluation.
3.2. Performance Degradation Evaluation Based on Condition Monitoring Information Fusion. The performance degradation for aero engines is described by condition monitoring data. Bayesian linear model (BLM) is proposed for fusing condition monitoring data to maximize the amount of meaning information. The advantages of BLM can be concluded as follows.

First, BLM can show the randomness of condition monitoring parameters and degradation degree variable.

Second, with BLM, the relevance can be considered to reduce the repetitive data.

Third, BLM can fuse data of different time by its learning function.

Last, with BLM, noise parameters can be designed to describe the uncertainty of measurement parameters.

Assuming that the performance degradation of aero engines can be described by performance monitoring matrix $\mathbf{X}=\left[\mathbf{X}_{1}, \mathbf{X}_{2}, \ldots, \mathbf{X}_{k}\right]$, where $k$ is the number of monitoring parameters, $\mathbf{X}_{k}$ is column matrix of $n$ row, and $n$ is monitoring number, the relation between performance degradation and monitoring parameters can be expressed by the following stochastic equation. Since there are some errors in most of monitoring parameters of aero engines, $e$ is introduced to express a monitoring error:

$$
\begin{gathered}
\mathbf{Y}=\mathbf{X} \boldsymbol{\theta}+\mathbf{e}, \\
\mathbf{e} \sim N\left(0, \sigma^{2}\right),
\end{gathered}
$$

where

$$
\mathbf{Y}=\left[\begin{array}{c}
y_{1} \\
y_{2} \\
\vdots \\
y_{n}
\end{array}\right], \quad \boldsymbol{\theta}=\left[\begin{array}{c}
\theta_{1} \\
\theta_{2} \\
\vdots \\
\theta_{n}
\end{array}\right], \quad \mathbf{e}=\left[\begin{array}{c}
e_{1} \\
e_{2} \\
\vdots \\
e_{n}
\end{array}\right]
$$

$\mathbf{e}_{i}$ is independent and assumed to obey normal distribution $N\left(0, \sigma^{2}\right)$ and $\sigma^{2}$ is known.

The mean $\mathbf{E}(\boldsymbol{\theta})$ and the covariance matrix $\mathbf{C}(\boldsymbol{\theta})$ of $\widehat{\boldsymbol{\theta}}$ can be computed by monitoring parameters and performance deterioration data according to (1).

For $\boldsymbol{\theta}$, the prior mean can be converted to posterior mean, given the observation matrix $\mathbf{X}$ Bayes mean square error matrix $\left(\mathbf{M}_{\hat{\theta}}\right)$ can be minimized by choosing ratio, which can be expressed as follows:

$$
\mathbf{M}_{\widehat{\boldsymbol{\theta}}}=E\left[(\boldsymbol{\theta}-\widehat{\boldsymbol{\theta}})(\boldsymbol{\theta}-\hat{\boldsymbol{\theta}})^{T}\right] .
$$

The estimator of linear minimum mean square error (LMMSE) can be got by (3).

In general, it is assumed that the monitoring parameters of aero engines obey inverse-Gauss distribution. The values of $\mathbf{E}(\boldsymbol{\theta})$ and $\mathbf{C}(\boldsymbol{\theta})$ can be learned by monitoring parameters of aero engines, which can be expressed as follows:

$$
\begin{aligned}
& \mathbf{E}(\theta \mid x, y)=\mu_{\theta}+\mathbf{C}(\theta) X^{T}\left(\mathbf{X C}(\theta) \mathbf{X}^{T}+\mathbf{C}_{e}\right)^{-1}\left(y-\mathbf{X} \mu_{\theta}\right), \\
& \mathbf{C}(\theta \mid x, y)=\mathbf{C}(\theta)-\mathbf{C}(\theta) \mathbf{X}^{T}\left(\mathbf{X C}(\theta) \mathbf{X}^{T}+\mathbf{C}_{e}\right)^{-1} \mathbf{X C}(\theta) .
\end{aligned}
$$

By applying (4) repeatedly, the monitoring data can be fused. 


\subsection{Reliability Modeling of Aero Engines}

3.3.1. Reliability Model of Aero Engines Based on Information Fusion. Failure modes of aero engines are generally classified into two types. One is catastrophic failures, in which aero engines break down as a result of some sudden external shocks; and the other is degradation failures, in which aero engines fail to function due to physical deterioration. Catastrophic failures and degradation failures are competing failures, both of which can influence reliability. In reality, catastrophic failures rarely happen. Only some information related to catastrophic failures can be gathered during inspection and repairing. In addition, there are interactions between catastrophic and degradation failures. On one hand, the degradation path of aero engines can be changed by catastrophic ones. On the other, the probability of catastrophic failures can be increased by degradation failures. Stochastic process shows the above reliability characteristics of aero engines. Gamma stochastic process is selected to describe reliability change of aero engines.

The gamma process with shape function $\alpha(t)>0$ and scale parameter $\beta>0$ is a continuous-time stochastic process. $\{Y(t), t \geq 0\}$ has the following properties:

(i) $Y(0)=0$;

(ii) $Y(\tau)-Y(t) \sim G a(\alpha(\tau)-\alpha(t), \beta)$, for all $\tau>t \geq 0$;

(iii) $Y(t)$ has independent increments.

The scale parameter is the measurement of intrinsic lifetime of aero engines, which shows that the catastrophic failure has effect on reliability. In general, scale parameter is invariable in one performance monitoring process. The event information can be used to estimate scale parameter. Shape function is changed with the performance degradation, which can be estimated according to the performance degradation result by using the method described in Section 3.2.

So, the event information and condition monitoring information can be fused by estimating shape function and scale parameter in gamma process.

The density function of degradation failures can be expressed as follows:

$$
f_{g}(y, \alpha(t), \beta)=\frac{\beta^{\alpha(t)}}{\Gamma(\alpha(t))} y^{\alpha(t)-1} e^{-\beta y},
$$

where $\Gamma$ is the gamma function given by $\Gamma(\alpha)=\int_{0}^{\infty} t^{\alpha-1} e^{-t} d t$.

Let shape parameter be proportional to expected degradation degree and time power, that is,

$$
\alpha(t)=k t^{v} .
$$

Further, (5) can be transformed as follows:

$$
f(y, \alpha(t), \beta)=\frac{\lambda^{\beta t^{\nu}}}{\Gamma\left(k t^{\nu}\right)} x^{k t^{\nu}-1} e^{-\beta y} .
$$

Based on the theory of system reliability, the reliability for degradation failures can be depicted as follows:

$$
R(t)=P\{T>t\} \Longrightarrow P\{y(t)<\xi\},
$$

where $\xi$ is the failure threshold for performance degradation of an aero engine. The degradation failure threshold indicates that the failure will be more possible when the degradation degree goes above the threshold. In the paper, the failure threshold is set as 0.2 on degradation degree deviation, the reasons are as follows.

First, degradation degree is the fusion of condition monitoring parameters. In the aero engines management, the deviation exceeding $20 \%$ of normal monitoring parameter is seen as abnormal.

Second, considering the relationship between performance degradation reliability and performance degradation degree, the degradation degree is set as 0.2 . And the risk can be controlled.

Then, the reliability evaluation for performance degradation of an aero engine can be depicted as follows:

$$
R(t)=\int_{0}^{\varepsilon} f_{w}(y) d y=\int_{0}^{\varepsilon} \frac{\lambda^{k t^{\nu}}}{\Gamma\left(k t^{\nu}\right)} x^{k t^{\nu}-1} e^{-\beta y} d y .
$$

3.3.2. The Estimation of Shape Parameter and Scale Parameter. It is easy to estimate shape parameter, which is the function of performance degradation degree and monitoring time. $\alpha(t)$ can be estimated by taking logarithms for (6) and using linear regression method.

Because the aero engine is high reliable system, there are rare sudden failures. The presented method put more emphasis on the influence of sudden failure on reliability than the prediction of sudden failure. The prior information is used to express scale parameter, which reflects that the sudden failure has effect on reliability. So, the reliability can be calculated according to the effect of sudden failure.

The catastrophic failure reliability evaluation can be transformed to estimate scale parameter $\beta$. It is assumed that the scale parameter $\beta$ has a conjugate gamma prior, that is,

$$
\pi(\beta \mid c, d)= \begin{cases}\frac{d^{c}}{\Gamma(c)} \beta^{c-1} e^{-d \beta}, & \text { if } \beta>0 \\ 0, & \text { if } \beta \leq 0\end{cases}
$$

The prior mean and variance of $\beta$ can be shown, respectively, as follows:

$$
\begin{aligned}
E(\beta) & =\frac{c}{d}, \\
\sigma^{2}(\beta) & =\frac{c}{d^{2}} .
\end{aligned}
$$

In the observed sample $\left\{\left(t_{1}, n_{1}\right),\left(t_{2}, n_{2}\right), \ldots,\left(t_{m}, n_{m}\right)\right\}, t_{i}$ is the happening time of catastrophic failure and $n_{i}$ is the number of catastrophic failure. Given the data and hyperparameters $c$ and $d$, posterior estimation of $\beta$ can be found as follows:

$$
\begin{gathered}
E\left(\beta^{\prime}\right)=\frac{c+\sum_{i=1}^{m} t_{i}}{d+\sum_{i=1}^{m} m_{i}}, \\
\sigma^{2}\left(\beta^{\prime}\right)=\frac{c+\sum_{i=1}^{m} t_{i}}{\left(d+\sum_{i=1}^{m} m_{i}\right)^{2}} .
\end{gathered}
$$


Hyperparameters $c$ and $d$ cannot be estimated directly. If the mean and variance of reliable lifetime $t_{R_{0}}$ can be estimated by using prior inspection and maintenance information under predetermined reliability, $\beta$ can be estimated by (13)

$$
\beta=\left[\frac{t_{R_{0}}}{\ln \left(1 / R_{0}\right)^{1 / r}}\right]^{1 / r} .
$$

If $\beta$ is known, $c$ and $d$ can be estimated by (11) The posterior mean and variance of $\beta$ can be calculated by (12).

\section{Maintenance Decision for Aero Engines}

4.1. Maintenance Decision Description for Aero Engines. An aero engine is assumed to be a partially observable system with gradually stochastically deterioration and sudden failure.

General assumptions on aero engines are as follows.

(i) It is considered that at time $t$, the reliability of aero engines can be expressed by $R(t)$.

(ii) When no action, such as repair or inspection, takes place, $R(t)$ is monotonic decreasing.

(iii) The aero engine is said to be "failed" if the $R(t)$ is below a given reliability threshold $R$. It means that the aero engine is no longer able to fulfill its functions in acceptable conditions, even if the aero engine is still functioning.

(iv) To get more information on the deterioration level of an aero engine and in order to be able to detect failures, the aero engine is perfectly monitored through periodic inspections. The aero engine is said to be "inspected" if the $R(t)$ is below a given inspection reliability threshold $R_{I}$. The time between two inspections, denoted by $\Delta t$, is called interinspection time.

It is deserving that the assumptions for aero engines can be used in other methods. In fact, complex repairable systems all follow the listed assumption in performance degradation process.

In engineering, the inspection can get more information by lubricating oil consumption, endoscope detection, magnetic blocking inspection, and so forth. Maintenance can be used as a tool for renewing reliability, which includes removal and shop visit. By inspection and maintenance, the condition of aero engines can be obtained, which can be used to give prior information of reliability evaluation.

The maintenance policy is driven by the knowledge of the aero engine state after inspection. At every inspection time, $\left(T_{n}\right)_{n \in N}, T_{0}=0$, two decisions have to be made.

(i) Determine whether the aero engine should be repaired preventively, or whether the aero engine should be left as it is.

(ii) Determine the next inspection.

The following maintenance decision rule is adopted. (i) If $R<R(t) \leq R_{I}$, the aero engine is still functioning, but deterioration accelerates, and then an inspection is performed and an inspection cost $C_{p}$ is incurred. If a maintenance action is taken, the reliability growth of the aero engine can be achieved.

(ii) If $R_{I} \leq R(t)$, then the aero engine is unchanged.

In the above two cases, $\Delta T_{n}$ is chosen by

$$
\Delta T_{n}=m(R(t))
$$

$m(\cdot)$ is interinspection time and inspection scheduling function. $\Delta T_{n}$ is inter-inspection time between inspection $i$ th and $(i+1)$ th.

Then, the time of the next scheduled inspection is

$$
T_{n+1}=T_{n}+\Delta T_{n}
$$

4.2. Maintenance Decision Model for Aircraft Engines. Within the maintenance structure, the two decision variables, the inspection threshold $R_{I}$ and inspection scheduling function $m(\cdot)$, are optimized in order to minimize the maintenance cost. $R_{I}$ is the function of $\xi_{I}$ (the performance degradation threshold). For the model, the optimization procedure aims at finding a couple $\left(\xi_{I}, m\right)$, on an infinite horizon, the cost of inspections, and $\xi_{I}$ balances the cost caused by failures and unavailability. A mathematical model of this maintenance decision and its associated cost is developed as follows.

The objective is to minimize the long run maintenance cost of time, which can be defined as

$$
E C_{\infty}=\frac{1}{\Delta t}\left(c_{i} P_{i}+c_{p} P_{p}+c_{c} P_{c}+c_{d} P_{d}\right)
$$

where $c_{d}$ is the cost of "inactivity of the system" per unit of time, $c_{i}$ is the cost of per inspection, $c_{p}$ is the cost of preventive maintenance (PM), and $c_{c}$ is the cost of per corrective maintenance $(\mathrm{CM}) . P_{i}, P_{p}, P_{c}$ are the probability at steady state to have inspection, a preventive or a corrective replacement, respectively, during a unit time length $\Delta t . P_{d}$ is the probability at steady state for the system to be in the operating state during $\Delta t$.

The computation of the probabilities in the right hand in (16) requires the knowledge of the stationary probability density function of the system evolution under the given decision. Let $f$ be the stationary probability density function of the maintained aero engine state. Denoted by $I$ the event corresponds to an inspection by $\bar{I}$ its complement. Conditioning the probability density $f$ and applying the law of total probability leads to

$$
f(x)=f_{1}(x)+f_{2}(x),
$$

where $f_{1}(x)=f(x \mid I) P(I)$ and $f_{2}(x)=f(x \mid \bar{I}) P(\bar{I})$.

$f_{1}(x)$ is the stationary density function of the system state being equal to $x$, and an inspection is scheduled.

$f_{2}(x)$ is the stationary density function of the system state being equal to $x$, and no inspection is scheduled. 

by

The probabilities $P_{i}, P_{p}, P_{c}$, and $P_{d}$ are, respectively, given

$$
\begin{aligned}
& P_{i}=\int_{0}^{\xi} f_{1}(x) d x, \\
& P_{p}=\int_{\xi}^{L} f_{1}(x) d x, \\
& P_{c}=\int_{L}^{+\infty} f(x) d x, \\
& P_{p}=\int_{0}^{\xi} f(x) d x .
\end{aligned}
$$

Then (16) can be expressed as follows:

$$
\begin{aligned}
& E C_{\infty}\left(\xi_{1}, \xi_{2}, \ldots, \xi_{N}\right) \\
&=\frac{1}{\Delta t}\left(c_{i} \int_{0}^{\xi} f_{1}(x) d x+c_{p} \int_{\xi}^{L} f_{1}(x) d x\right. \\
&\left.\quad+c_{c} \int_{L}^{+\infty} f(x) d x+c_{d} \int_{0}^{\xi} f(x) d x\right) .
\end{aligned}
$$

4.3. Maintenance Optimization by Partial Swarm Optimization for Aero Engines. The partial swarm optimization (PSO) is a stochastic global optimization method originally proposed by Eberhart and Kennedy in 1995 [15], which is based on social behavior simulation. PSO is similar to evolutionary computation techniques in that a population of potential solutions to the problem under consideration is used to probe the search space. However, in PSO, each individual of the population has an adaptable velocity (position change), according to which it moves in the search space. Moreover, each individual has a memory, remembering the best position of the search space it has ever visited. Thus, its movement is an aggregated acceleration towards its best previously visited position and towards the best individual of a topological neighborhood. Since the term "acceleration" was mainly used for partial systems in Particle Physics, the pioneers of this technique decided to use the term particle for each individual, and the name swarm for the population, which comes up with the name Particle Swarm for their algorithm [15]. The PSO has the advantages of easy understanding, simple operation, and rapid searching. A typical PSO generates an initial population randomly. Two factors including position and velocity characterize a particle status in a search space, as follows:

$$
\begin{aligned}
\mathbf{V}_{i}^{\text {new }}= & \omega \times \mathbf{V}_{i}+c_{1} \times \operatorname{rand}_{1} \times\left(\mathbf{P}_{i}-Z_{i}\right) \\
& +c_{2} \times \operatorname{rand}_{2} \times\left(\mathbf{P}_{g}-Z_{i}\right) Z_{i}^{\text {new }} \\
= & Z_{i}+V_{i}^{\text {new }},
\end{aligned}
$$

where $\mathbf{V}_{i}^{\text {new }}$ and $\mathbf{V}_{i}$ represent the updated velocity and current velocity vector in a search for the $i$ th particle, $Z_{i}$ represents the current position vector in a search space; $\mathbf{P}_{i}$ and $\mathbf{P}_{g}$ are currently the particle best solution and global solution;
$\mathbf{P}_{i}-Z_{i}$ reveals the distance between $\mathbf{P}_{i}$ and $Z_{i}$; and $\mathbf{P}_{g}-$ $Z_{i}$ is the distance between $\mathbf{P}_{g}$ and $Z_{i}$; rand1 and rand2 are two random functions with a range $[0,1] . c_{1}$ and $c_{2}$ are positive constant parameters called acceleration coefficients controlling the movement steps of particles. $Z_{i}^{\text {new }}$ and $Z_{i}$ represent the updated position and current position of the $i$ th particles. The $\omega$ is an inertia weight and controls, with $c_{1}$ and $c_{2}$, the effect of previous values of particles velocity on next one. The update procedure consecutively iterates until a predetermined terminal condition is reached. The best solution is thereby obtained.

The steps of maintenance optimization by PSO for aero engines can be described as follows.

Step 1 (establish the superior initial particle population). Randomly generate inspection and maintenance periods. Accordingly, the initial particle population can be established.

Step 2 (determine the current local and global optimums). For each particle, select the particle with the lowest maintenance cost in previous iterations as a current local optimum. Then, select the particle with the lowest maintenance cost from previous iterations as the current global optimum.

Step 3 (update the current velocity and position of particles). For each particle, one can derive the updated velocity given parameter values of $\omega, c_{1}$, and $c_{2}$ by putting the current velocity, current particle position, local optimum; and global optimum, the updated particle position is then determined by putting the updated velocity and current particle position. Furthermore, the elitist conservative strategy is applied, so that the best particle in the course of iterations is conserved to guide the search of other particles during each iteration.

Step 4 (get the maintenance optimization result). Terminate the PSO and output optimized maintenance periods of aero engines when the total number of iterations passes a predetermined value or when fitness does not increase in continuously maximum iterations.

Step 5 (adaptive maintenance optimization). After the inspection, more information can be obtained to describe the reliability of aero engines, and then the inspection and repair can be adaptively adjusted. Uncertainty about the parameters of reliability is described by prior distribution, and the existing distribution is updated by using Bayesian method. For shape parameter $\alpha(t)$, BLM can be used for parameter learning. For the scale parameter $\beta$, the parameter can be updated by applying Bayesian methods using inspection, repair, and failure information. Then, the parameter about the uncertainties can be reduced to some extent. The change of performance degradation path and failure law can be found through shape and scale parameters learning. If there are no virtual changes, the maintenance decision can be seen as realistic. If there are some changes, the maintenance decision should be adjusted and Steps 1 to 4 should be repeated. 
TABLE 1: Key performance monitoring parameters for some aero engines.

\begin{tabular}{|c|c|c|c|c|c|c|c|c|}
\hline $\mathrm{NO}$ & DEGT & DWF & DOP & DHPRS & DLPRV & DHPRV & TSI (FH) & PDD \\
\hline 1 & 7.51 & 2.54 & 1.89 & -7.27 & 1.06 & 0.32 & 4055 & 0.1192 \\
\hline 2 & -4.74 & 3.52 & 1.92 & -5.16 & 0.52 & 0.55 & 7095 & 0.0459 \\
\hline 3 & -0.03 & 2.03 & 1.19 & -8.33 & 0.57 & 0.37 & 7801 & 0.0378 \\
\hline 4 & 8.04 & 5.16 & 1.69 & -7.74 & 0.24 & 0.57 & 3331 & 0.1176 \\
\hline 5 & 7.77 & 7.80 & 2.12 & -6.81 & 0.86 & 0.46 & 3832 & 0.1308 \\
\hline$\vdots$ & $\vdots$ & $\vdots$ & $\vdots$ & $\vdots$ & $\vdots$ & $\vdots$ & $\vdots$ & $\vdots$ \\
\hline 31 & 22.69 & 7.58 & 2.12 & -1.07 & 0.64 & 0.99 & 1055 & 0.2000 \\
\hline 32 & 4.23 & 4.83 & 1.96 & -58.92 & 0.17 & 0.62 & 3397 & 0.0996 \\
\hline 33 & 14.28 & 5.25 & 1.63 & -2.03 & 0.78 & 0.94 & 1422 & 0.1572 \\
\hline 34 & 11.38 & 3.14 & 1.63 & 4.19 & 0.23 & 0.74 & 1830 & 0.1465 \\
\hline 35 & 8.24 & 3.17 & 2.18 & 9.78 & 1.05 & 0.76 & 1954 & 0.1185 \\
\hline
\end{tabular}

TABLE 2: Key performance monitoring parameters for one aero engine.

\begin{tabular}{|c|c|c|c|c|c|c|c|}
\hline $\mathrm{NO}$ & DEGT & DWF & DOP & DHPRS & DLPRV & DHPRV & TSI (FH) \\
\hline 1 & -4.5379 & 5.3806 & 1.7040 & 0.2000 & 1.0000 & -4.5379 & 161 \\
\hline 2 & -3.5419 & 5.3720 & 1.8987 & 0.2080 & 1.0164 & -3.5419 & 210 \\
\hline 3 & 5.4312 & 5.7129 & 2.0777 & 0.1320 & 0.9238 & 5.4312 & 528 \\
\hline 4 & 10.3950 & 5.9406 & 2.2084 & 0.0990 & 0.9303 & 10.3950 & 619 \\
\hline 5 & 10.7172 & 5.9749 & 1.9809 & 0.1299 & 0.9964 & 10.7172 & 871 \\
\hline : & $\vdots$ & $\vdots$ & $\vdots$ & $\vdots$ & $\vdots$ & $\vdots$ & $\vdots$ \\
\hline 20 & 9.4043 & 6.2813 & 2.2450 & 1.0450 & 1.1325 & 9.4043 & 3998 \\
\hline 21 & 8.2111 & 6.3240 & 2.1250 & 1.0939 & 1.1693 & 8.2111 & 4213 \\
\hline 22 & 11.7701 & 6.4844 & 2.1064 & 1.0691 & 1.1255 & 11.7701 & 4428 \\
\hline 23 & 9.8428 & 6.2183 & 2.0891 & 0.9079 & 1.1342 & 9.8428 & 4644 \\
\hline 24 & 3.3712 & 5.4088 & 2.0831 & 0.6547 & 1.1284 & 3.3712 & 4851 \\
\hline
\end{tabular}

\section{Examples}

Table 1 shows 35 samples of repaired or replaced engines. There are six parameters that have been monitored, which are the deviation exhaust gas temperature (DEGT), the deviation of fuel flow (DWF), the deviation of oil pressure (DOP), the deviation of high pressure rotor speed (DHPRS), the deviation of the low pressure rotor vibration value (DLPRV), and the deviation of high-pressure rotor vibration value (DHPRV). From the beginning of the monitoring moment, the engines' time since installation (TSI) can be obtained. From the data of Table 1, the relationship between the various monitoring parameters can be extracted and be used as the basis for information fusion. Table 2 shows six monitoring parameters and TSI of one aero engine for 24 months. The data is collected every month.

Figure 2 shows performance deviation for some aircraft engine based on multiparameters monitoring. Based on the monitoring parameters from Table 1, the relationship between PDD and performance monitoring parameters can be obtained by using the algorithm proposed in Section 3.1. The vector fused monitoring parameter is $\widehat{\theta}=$ $[-0.0103,0.1795,0.0016,0.0318,0.0152,-0.0158,0.0156]^{T}$. The estimated values of performance degradation through fusion and values of actual performance degradation are compared in Figure 3. Figure 3 shows that the PDD predictive value corresponded to the real value, which demonstrates the accuracy of BLM. In Figure 3, the real performance degradation degree (PDD) is not the data collected directly, instead obtained through Monte-Carlo simulation method, in the case of a given reliability of $90 \%$, and in accordance with the case that its performance degradation meets gamma random process.

Comparing Figure 3 with Figure 2, we can conclude the following.

(i) BLM can actually represent more parameters of monitoring information, which reflects the advantages of information fusion.

(ii) The predictive value corresponds to the real value, which shows the accuracy of BLM.

With the calculation of $\hat{\theta}$ as an input variable, together with the monitoring parameters in Table 2, the performance degradation degree can be predicted for monitoring aero engines by (4). Using the consecutive monitoring data in Table 2, the shape parameter $\alpha(t)$ of gamma process can be estimated according to (6). $\alpha(t)>1$ shows that the aero engine is the performance degradation. Figure 4 shows the relationship $\alpha$ and $R(t)$ in the performance degradation process, while $\beta$ is constant. 


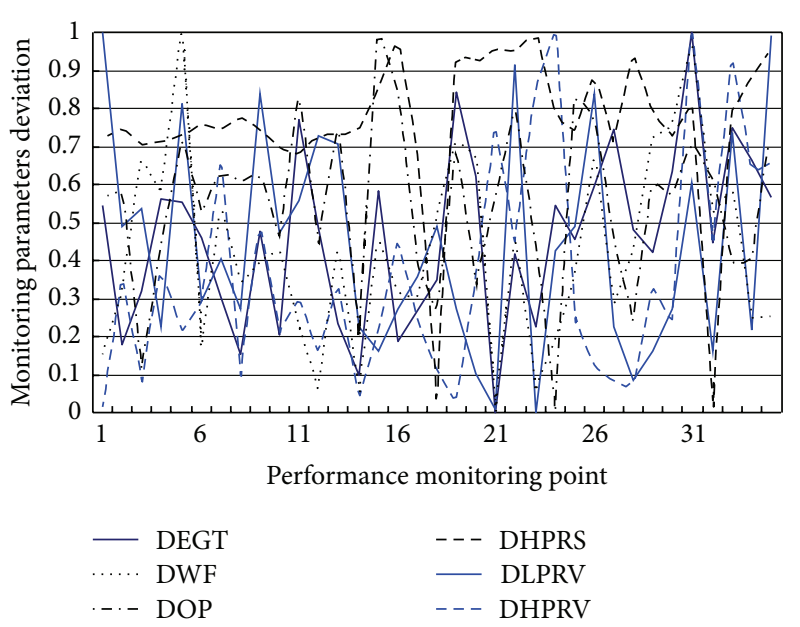

FIgURE 2: Performance deviation for some aircraft engine based on multiparameters monitoring.

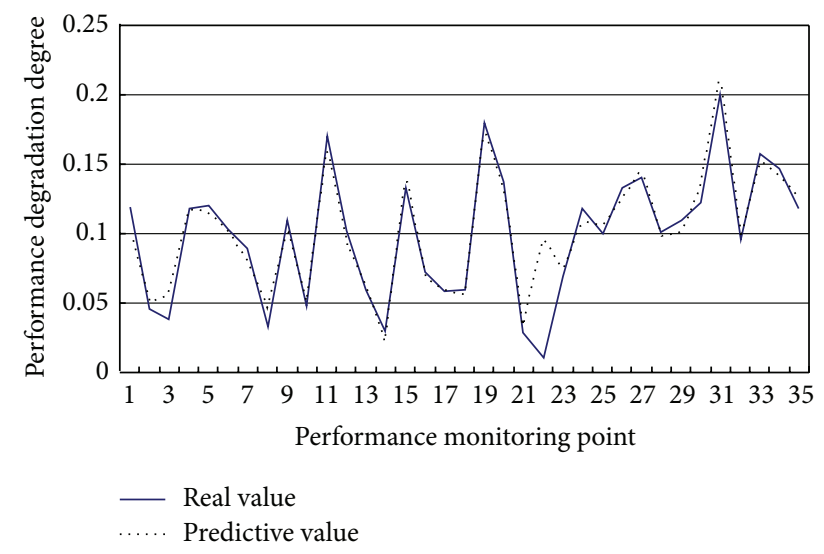

FIGURE 3: The difference of performance degradation between real value and predictive value.

In fact, there is no inspection and repair information about the new aero engines. The scale parameter $\beta$ can be estimated by analyzing design parameters and running condition as prior information. The prior value of sudden failures can be determined as $E\left(R_{2500}\right)=0.965$, and $E(\beta)$ can be computed by (11). The posterior mean and variance of $\widehat{\beta}$ can be computed by (12).

Figure 5 shows the relationship $\beta$ and $R(t)$, while $\alpha$ is constant.

Take the estimation of $\widehat{\alpha}(t)$ and $\widehat{\beta}$ in (18), $P_{i}, P_{p}, P_{c}$, and $P_{d}$ can be calculated respectively. The difference between $f_{1}(x)$ and $f_{2}(x)$ is the scale parameter updated by inspection information.

Take $P_{i}, P_{p}, P_{c}$, and $P_{d}$ in (19), which is the optimization function of maintenance decision for aero engines. In (19), $C_{d}=10$ thousand dollar, $C_{i}=50$ thousand dollar, $C_{p}=$ 200 thousand dollar, $C_{c}=500$ thousand dollar, and the optimization time span is $10000 \mathrm{FH}$.

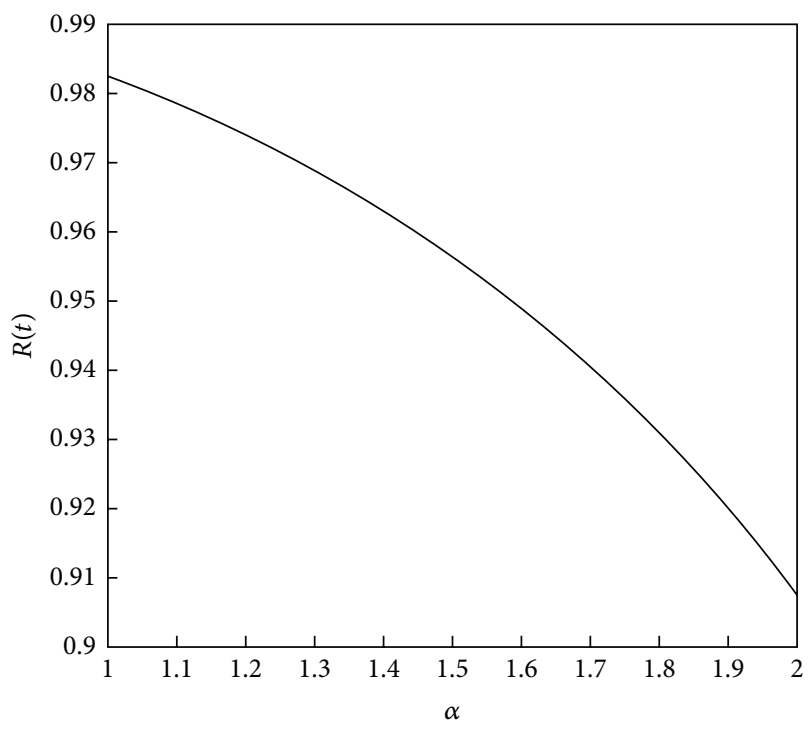

FIGURE 4: The relationship $\alpha$ and $R(t)$ in the performance degradation process.

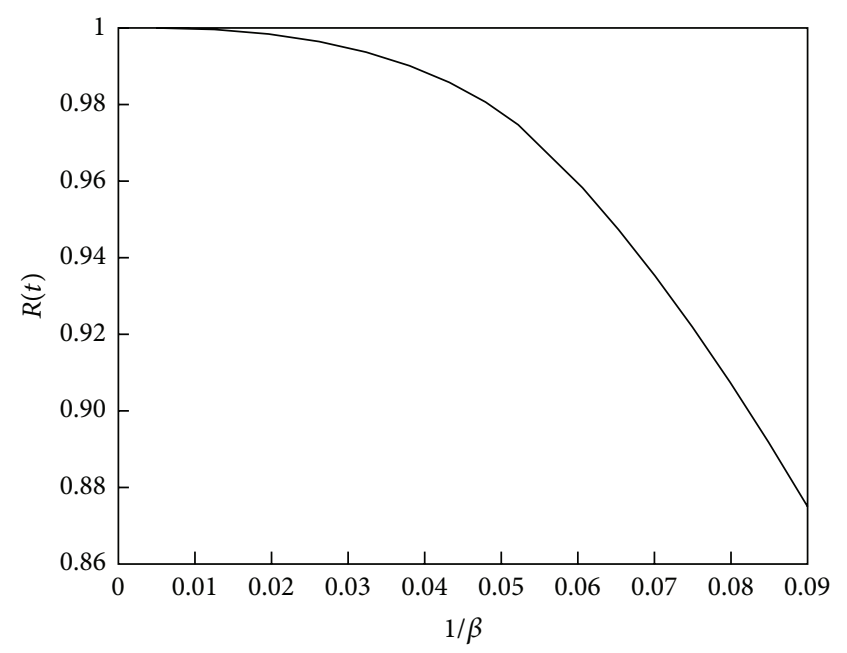

FIgURE 5: The relationship between $\beta$ and $R(t)$.

The PSO for maintenance decision optimization is then applied under an initial population of 30 particles. Six experiments have been made. Learning factor $C_{1}$ is 1.2 , learning factor $C_{2}$ is 1.2 , and inertia weight $\omega$ is from 0.3 to 0.8 . The stopping criterion is the number of iterations equal to 1000 . When the iteration time is 520 , the maintenance cost has been optimized The maintenance optimization by PSO is shown as Figure 6.

The optimization results of maintenance decision for aero engines by PSO are shows in Table 3. There are two inspections and one repair during 10000FH.

In engineering, the inspection and maintenance can be made by condition-based decision in engineering. In reliability management of aero engines, single parameter and multi-parameter can be used for monitoring condition. In single parameter method, DEGT exceeding $20 \%$ can be seen 


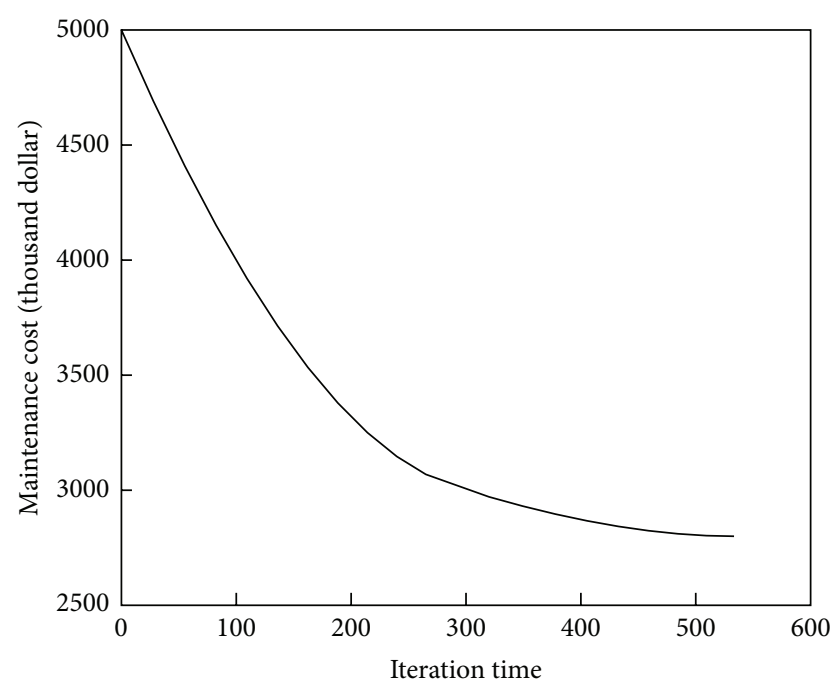

FIGURE 6: The maintenance optimization by PSO.

TABlE 3: The maintenance optimization results.

\begin{tabular}{lcccc}
\hline No. & Action & Inspection threshold & $t$ & $R$ \\
\hline 1 & Inspection & 0.1615 & 3908 & 0.9415 \\
2 & Repair & - & 5709 & 0.9029 \\
3 & Inspection & 0.1428 & 8962 & 0.9407 \\
\hline
\end{tabular}

TABLE 4: The maintenance decision in engineering.

\begin{tabular}{lcc}
\hline No. & Action & $t$ \\
\hline 1 & Inspection & 4102 \\
2 & Repair & 5521 \\
3 & Inspection & 8735 \\
4 & Repair & 9679 \\
\hline
\end{tabular}

as inspection or maintenance criterion. DEGT and DWF are used for multiparameter monitoring; the multiparameter expression is $0.75 \times \mathrm{DEGT}+0.25 \times \theta \times \mathrm{DWF}$, which, if exceeding $20 \%$, can be seen as inspection or maintenance criterion. It is deserving that DEGT and DWF are used to express normalization value. $\theta$ is different from category of aero engines. In the paper, the value of $\theta$ is 9 . Multiparameters method is used for comparison in the paper; the results are shown in Table 4. By comparison, the maintenance decision can be optimized by reducing time of inspection and maintenance. The objective of reducing maintenance cost has been realized under the premise of controlling risk.

\section{Conclusions}

In this paper, a novel maintenance decision method based on information fusion is presented. The characteristics can be summed up as follows. First, the performance degradation degree of aero engines is calculated through fusing condition monitoring information based on BLM. Second, the reliability evaluation of aero engines is built on gamma stochastic process, which fuses the performance degradation degree results and event information by shape and scale parameter estimation. Third, the maintenance optimization for aero engines based on real-time reliability evaluation is developed, and the objective of reducing maintenance cost and risk is realized through the optimization of performance degradation threshold and maintenance interval.

\section{Acknowledgments}

This work was supported by National Natural Science Foundation and Aviation Fund of China 60879001 and U1233115, and Qinglan Project.

\section{References}

[1] A. K. S. Jardine, D. Lin, and D. Banjevic, "A review on machinery diagnostics and prognostics implementing condition-based maintenance," Mechanical Systems and Signal Processing, vol. 20, no. 7, pp. 1483-1510, 2006.

[2] J. Bell, Condition Based Maintenance Plus DoD Guidebook, Department of Defense, 2008.

[3] G. Niu, B. S. Yang, and M. Pecht, "Development of an optimized condition-based maintenance system by data fusion and reliability-centered maintenance," Reliability Engineering and System Safety, vol. 95, no. 7, pp. 786-796, 2010.

[4] A. K. S. Jardine, P. M. Anderson, and D. S. Mann, "Application of the Weibull proportional hazard model to aircraft and marine engine failure data," Quality and Reliability Engineering International, vol. 3, pp. 77-82, 1987.

[5] P. J. Vlok, M. Wnek, and M. Zygmunt, "Utilising statistical residual life estimates of bearings to quantify the influence of preventive maintenance actions," Mechanical Systems and Signal Processing, vol. 18, no. 4, pp. 833-847, 2004.

[6] C. Bunks, D. McCarthy, and T. Al-Ani, "Condition-based maintenance of machines using hidden Markov models," Mechanical Systems and Signal Processing, vol. 14, no. 4, pp. 597-612, 2000.

[7] M. Dong and D. He, "Hidden semi-markov models for machinery health diagnosis and prognosis," Transactions of the North American Manufacturing Research Institution of SME, vol. 32, pp. 199-206, 2004.

[8] D. Lin and V. Makis, "Recursive filters for a partially observable system subject to random failure," Advances in Applied Probability, vol. 35, no. 1, pp. 207-227, 2003.

[9] W. Wang, "A model to predict the residual life of rolling element bearings given monitored condition information to date," IMA Journal Management Mathematics, vol. 13, no. 1, pp. 3-16, 2002.

[10] J. Vatn, P. Hokstad, and L. Bodsberg, "An overall model for maintenance optimization," Reliability Engineering and System Safety, vol. 51, no. 3, pp. 241-257, 1996.

[11] C. G. Vassiliadis and E. N. Pistikopoulos, "Maintenance scheduling and process optimization under uncertainty," Computers and Chemical Engineering, vol. 25, no. 2-3, pp. 217-236, 2001.

[12] T. Aven and I. T. Castro, "A minimal repair replacement model with two types of failure and a safety constraint," European Journal of Operational Research, vol. 188, no. 2, pp. 506-515, 2008.

[13] J. Vatn and T. Aven, "An approach to maintenance optimization where safety issues are important," Reliability Engineering and System Safety, vol. 95, no. 1, pp. 58-63, 2010. 
[14] A. Sanchez, S. Carlos, S. Martorell, and J. F. Villanueva, "Addressing imperfect maintenance modelling uncertainty in unavailability and cost based optimization," Reliability Engineering and System Safety, vol. 94, no. 1, pp. 22-32, 2009.

[15] R. Eberhart and J. Kennedy, "A new optimizer using particle swarm theory," in Proceedings of the 6th International Symposium on Micro Machine and Human Science, pp. 39-43, IEEE Service Center, Piscataway, NJ, USA, October 1995. 


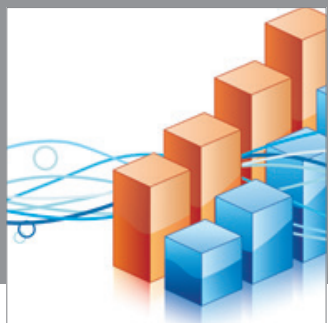

Advances in

Operations Research

mansans

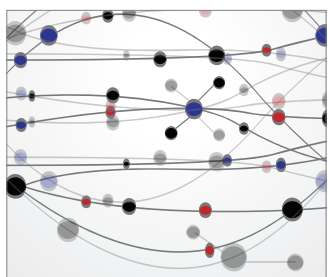

The Scientific World Journal
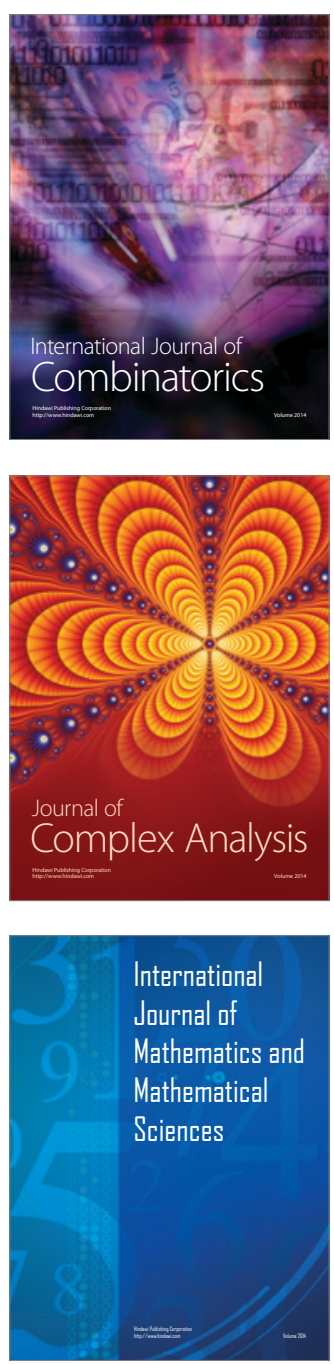
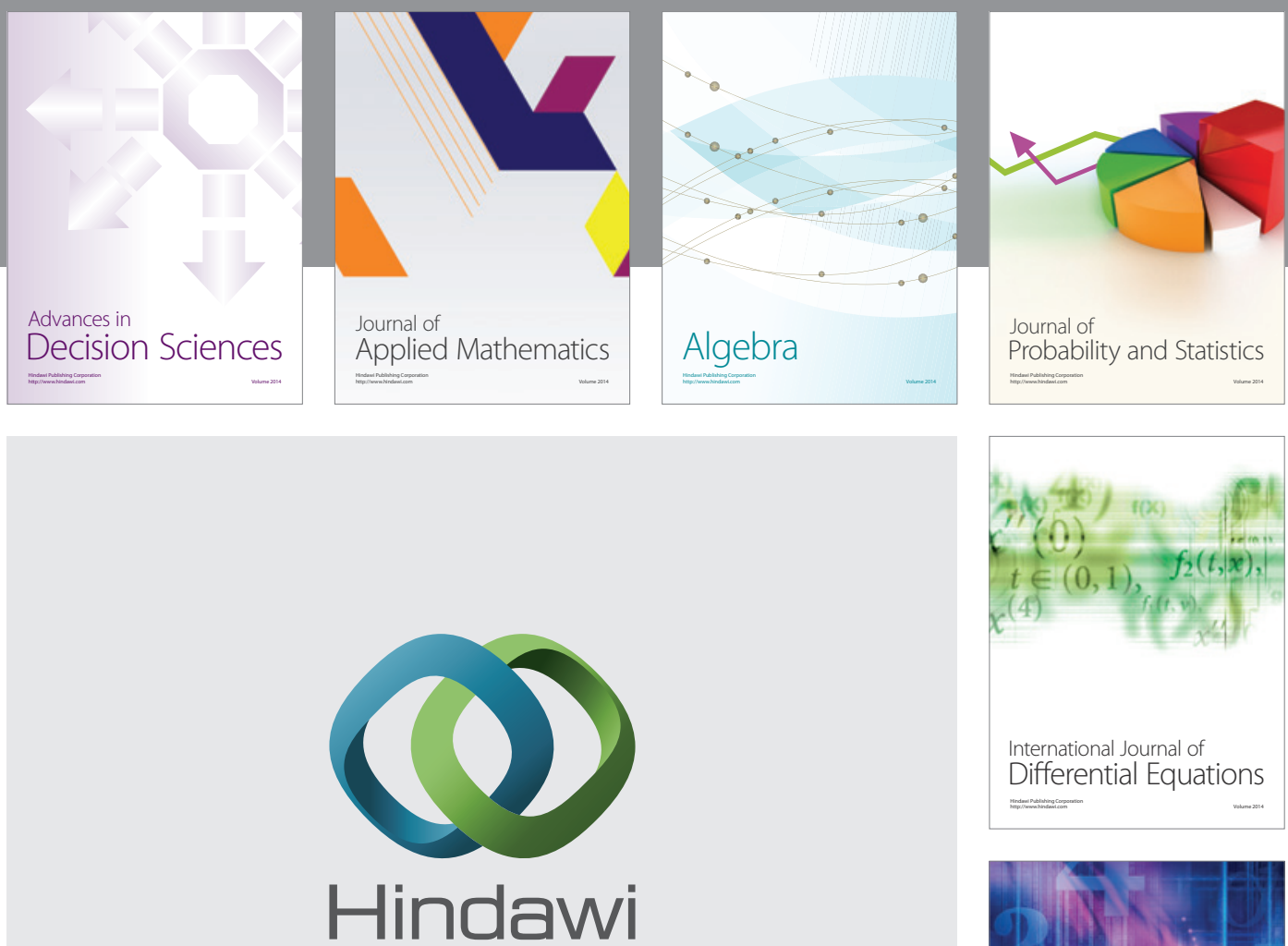

Submit your manuscripts at http://www.hindawi.com
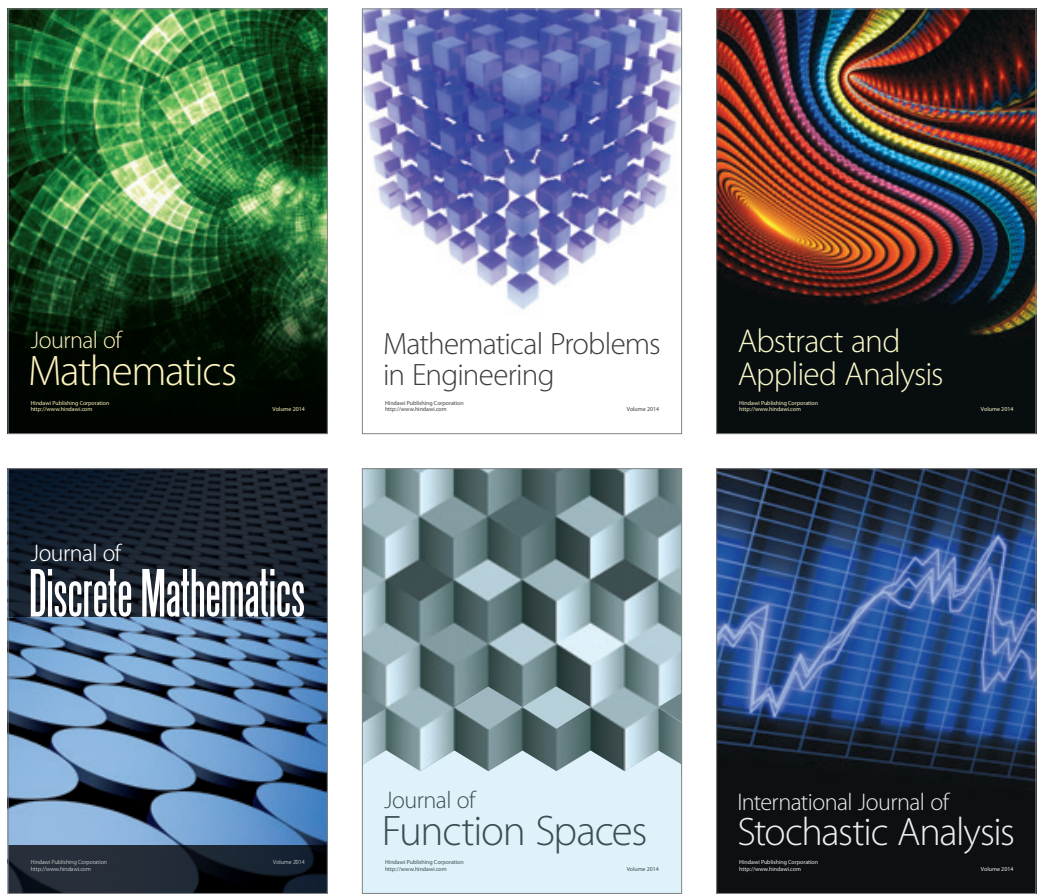

Journal of

Function Spaces

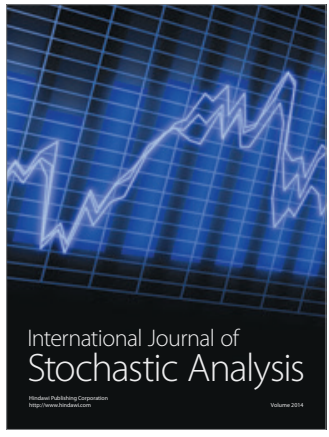

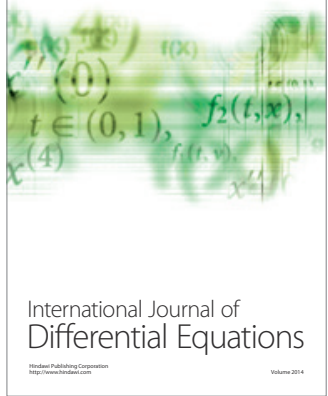
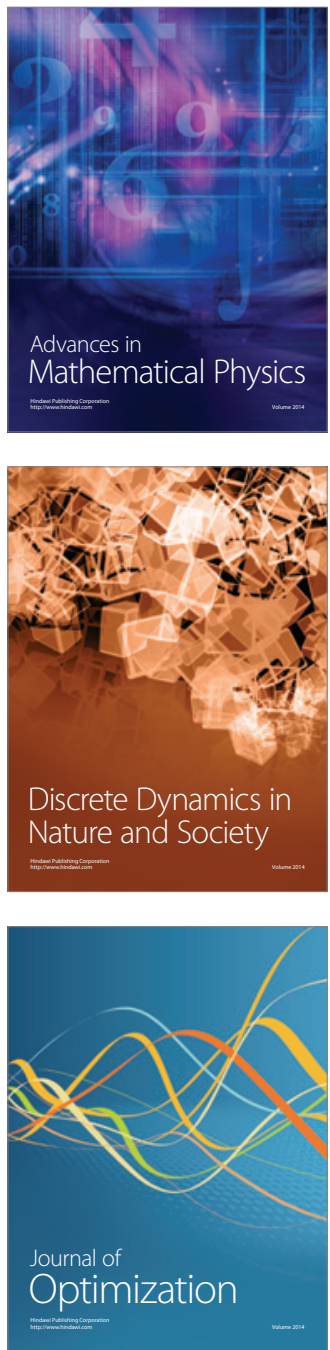